\title{
Concentration-Dependent Conformational \\ Isomerization of Fluorenone-Based Polycatenar in 2D Polymorphic Self-Assembly
}

Bang Li, ${ }^{\mathrm{a},{ }^{\ddagger}}$ Yuantao Liu, ${ }^{\mathrm{b}, \downarrow}$ Yuan Tao, ${ }^{\mathrm{b}}$ Yi Wang, ${ }^{\mathrm{a}}$ Xinrui Miao, ${ }^{\mathrm{a}, *}$ Xiaohong Cheng, ${ }^{\mathrm{b}, *}$ and Wenli

$\operatorname{Deng}^{\mathrm{a}, *}$

†College of Materials Science and Engineering, South China University of Technology, Guangzhou 510640, People's Republic of China

${ }^{\ddagger}$ Key Laboratory of Medicinal Chemistry for Natural Resources, Chemistry School of Chemical Science and Technology, Yunnan University, Kunming 650091, People’s Republic of China

Corresponding authors: Xinrui Miao (msxrmiao@scut.edu.cn)

Xiaohong Cheng (xhcheng@ynu.edu.cn)

Wenli Deng (wldeng@scut.edu.cn)

$\S$ These authors contributed equally to this work. 


\section{Synthesis and analytical data}

Reactions requiring an inert gas atmosphere were conducted under argon and the glassware was oven-dried $\left(140^{\circ} \mathrm{C}\right)$. Tetrahydrofuran (THF) was distilled from sodium prior to use. Commercially available chemicals were used as received. ${ }^{1} \mathrm{H}$ NMR and ${ }^{13} \mathrm{C}$ NMR spectra were recorded on a Bruker-DRX-300 spectrometer and a Bruker-DRX-400 spectrometer. Elemental analysis was performed using an Elementar VARIO EL elemental analyzer. Thin-layer chromatography was performed on aluminum plates precoated with 5735 silica gel 60 PF254 (Merck). Column chromatography was performed on Merck silica gel 60 (230-400 mesh). HRMS were performed on LTQ Orbitrap XL. Compound $\mathbf{1}^{\mathrm{S} 1}$, compound $\mathbf{4}^{\mathrm{S} 2 \mathrm{~S} 3}$ and compound $\mathbf{6}^{\mathrm{S} 4}$ were individually synthesized according to our previously published procedures.

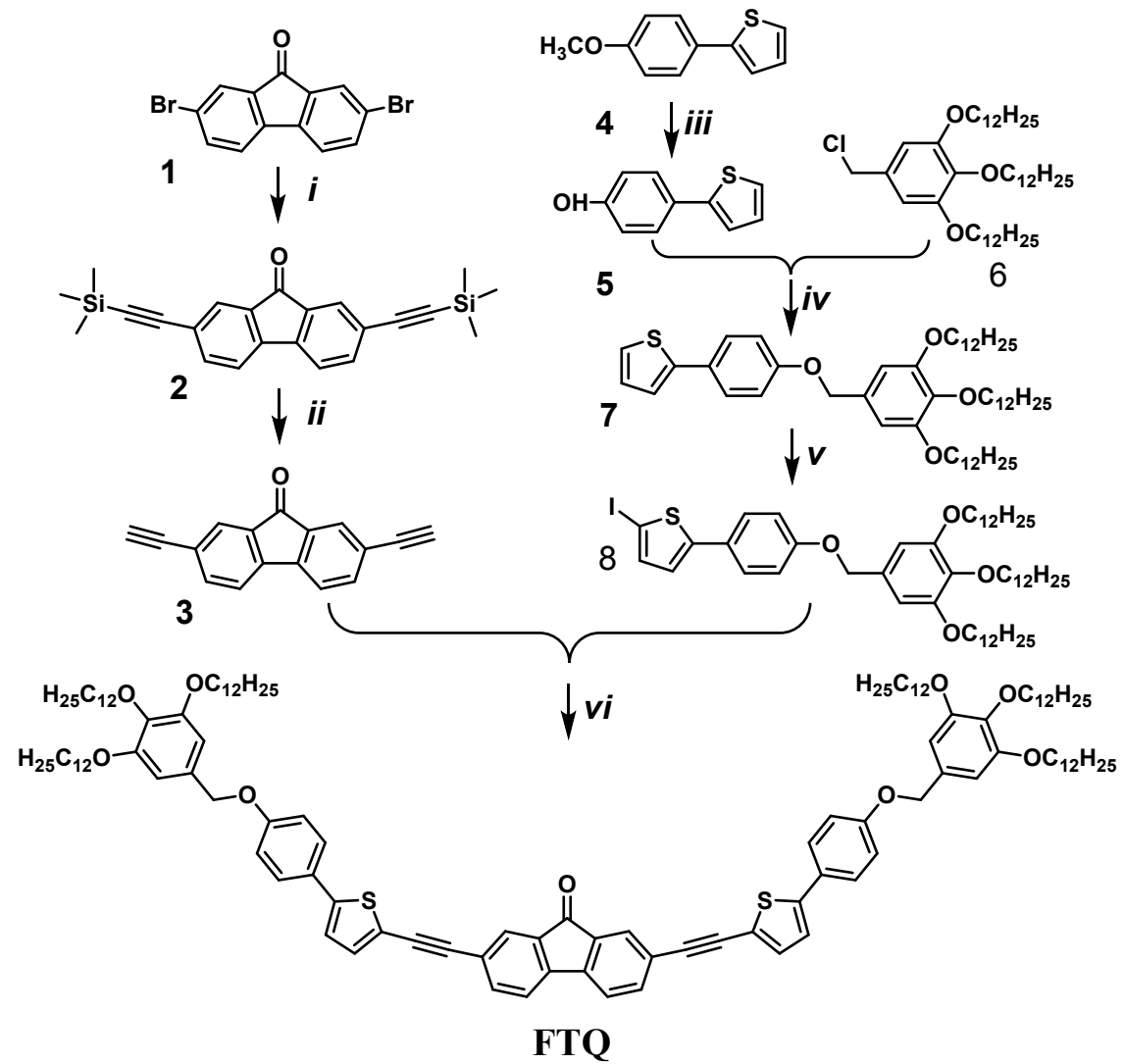

Scheme S1: Synthesis of FTQ. Reagents and conditions: i) trimethylsilyne, $\mathrm{CuI}, \mathrm{Pd}\left(\mathrm{PPh}_{3}\right)_{2} \mathrm{Cl}_{2}, \mathrm{Et}_{3} \mathrm{~N}, \mathrm{THF}$, $\mathrm{N}_{2}, 50{ }^{\circ} \mathrm{C}, 15 \mathrm{~h}$; ii) THF, $\mathrm{MeOH}, \mathrm{K}_{2} \mathrm{CO}_{3}, \mathrm{~N}_{2}, \mathrm{RT}, 4 \mathrm{~h}$; iii) $\mathrm{BBr}_{3}, \mathrm{CH}_{2} \mathrm{Cl}_{2}, \mathrm{RT}, 12 \mathrm{~h}$; iv) $\mathrm{K}_{2} \mathrm{CO}_{3}, \mathrm{DMF}, \mathrm{N}_{2}$, $90{ }^{\circ} \mathrm{C}, 12 \mathrm{~h}$; v) NIS, $\mathrm{CH}_{2} \mathrm{Cl}, \mathrm{CH}_{3} \mathrm{COOH}, \mathrm{RT}, 12 \mathrm{~h}$; vi) $\mathrm{Pd}\left(\mathrm{PPh}_{3}\right)_{2} \mathrm{Cl}_{2}, \mathrm{Et}_{3} \mathrm{~N}, \mathrm{THF}, \mathrm{N}_{2}, 50{ }^{\circ} \mathrm{C}$.

\section{Compound 2:}

A mixture of compound 1 (338 mg, $1 \mathrm{mmol})$ and trimethylsilyne $(216 \mathrm{mg}, 2.2 \mathrm{mmol})$ was dissolved in dry 
THF (10 mL) and dry triethylamine $(5 \mathrm{~mL})$ under a nitrogen atmosphere. Then $\mathrm{Pd}\left(\mathrm{PPh}_{3}\right)_{2} \mathrm{Cl}_{2}(15 \mathrm{mg}), \mathrm{CuI}(30$ $\mathrm{mg}, 0.16 \mathrm{mmol}$ ) was added, and the mixture was refluxed at $50{ }^{\circ} \mathrm{C}$ for $15 \mathrm{~h}$. After the reaction was complete (TLC), ethyl acetate $(20 \mathrm{~mL})$ was added, and the mixture was washed with brine $(3 \times 20 \mathrm{~mL})$ and dried over anhydrous $\mathrm{Na}_{2} \mathrm{SO}_{4}$. The solvent was evaporated in vacuo. The residue was purified by column chromatography (petroleum ether : Methylene chloride $=3: 1$ ). Yield: $205 \mathrm{mg}, 55 \%$; yellow solid. ${ }^{1} \mathrm{H}$ NMR (400 MHz, $\mathrm{CDCl}_{3}$ ), $\delta$ (ppm): 7.73 (s, $2 \mathrm{H}$, fluorenone-1,8H), 7.59-7.57 (dd, $J=1.2 \mathrm{~Hz}, J=7.6 \mathrm{~Hz}, 2 \mathrm{H}$, fluorenone-3,6H), 7.46-7.44 (d, $J=8 \mathrm{~Hz}, 2 \mathrm{H}$, fluorenone-4,5H), 1.09 (s, $18 \mathrm{H} 6 \mathrm{SiCH}_{3}$ ).

\section{Compound 3:}

Compound 2 (205 mg $0.54 \mathrm{mmol})$ was dissolved in THF : $\mathrm{CH}_{3} \mathrm{OH}=2: 1(20 \mathrm{~mL}), \mathrm{K}_{2} \mathrm{CO}_{3}(372 \mathrm{mg}, 2.7$ mmol) was added and the mixture was stirred at room temperature for $4 \mathrm{~h}$. After the reaction was complete (TLC), ethyl acetate $(50 \mathrm{~mL})$ was added, and the mixture was washed with brine $(3 \times 20 \mathrm{~mL})$ and dried over anhydrous $\mathrm{Na}_{2} \mathrm{SO}_{4}$. The solvent was evaporated in vacuo. The residue was purified by column chromatography (petroleum ether : methylene chloride $=2: 1$ ). Yield: $117 \mathrm{mg}, 95 \%$; yellow solid. ${ }^{1} \mathrm{H}$ NMR (400 MHz, $\mathrm{CDCl}_{3}$ ), $\delta$ (ppm): 7.76 (s, $2 \mathrm{H}$, fluorenone-1,8H), 7.64-7.62 (dd, $J=1.6 \mathrm{~Hz}, J=8.0 \mathrm{~Hz}, 2 \mathrm{H}$, fluorenone-3,6H), 7.50-7.48 (d, $J=7.6 \mathrm{~Hz}, 2 \mathrm{H}$, fluorenone-4,5H), 3.1 (s, $2 \mathrm{H} 2$ alkyne-H)

\section{2-(4-methoxyphenyl)thiophene 4:}

4-methoxybenzene boronic acid (1.52 g, $10 \mathrm{mmol})$, 2-bromothiophene (1.64 g, $10 \mathrm{mmol})$ was dissolved in toluene : $\mathrm{H}_{2} \mathrm{O}=1: 1(30 \mathrm{~mL})$, then $\mathrm{Pd}\left(\mathrm{PPh}_{3}\right)_{4}(120 \mathrm{mg}), \mathrm{Na}_{2} \mathrm{CO}_{3}(2.8 \mathrm{~g}, 20 \mathrm{mmol})$ was added under $\mathrm{N}_{2}$. The reaction mixture was stirred for $30 \mathrm{~min}$ at room temperature, and then heated to reflux for $10 \mathrm{~h}$. The reaction mixture was then cooled to ambient temperature, extracted with EtOAc $(3 \times 30 \mathrm{ml})$ and washed with brine. The organic extracts were concentrated, and the resulting residue was purified by column chromatography (petroleum ether: ethyl acetate $=15: 1)$. Yield: $1.76 \mathrm{~g}, 93 \% ;{ }^{1} \mathrm{H} \mathrm{NMR}\left(400 \mathrm{MHz}, \mathrm{CDCl}_{3}\right), \delta$ (ppm): $\delta=7.53-7.51(\mathrm{~d}, J=6.8 \mathrm{~Hz}, 2 \mathrm{H}, 2 \mathrm{ArH}), 7.25-7.21(\mathrm{~m}, 2 \mathrm{H}, 2$ thiopheneH), 7.08-7.06 (m, $1 \mathrm{H}$, thiopheneH), 6.90-6.86 (d, $J=6.8 \mathrm{~Hz}, 2 \mathrm{H}, 2 \mathrm{ArH}), 3.83$ (s, $3 \mathrm{H}, \mathrm{CH}_{3}$ ).

\section{4-(thiophen-2-yl)phenol 5:}

2-(4-methoxyphenyl)thiophene 4 (224 mg, $1.18 \mathrm{mmol})$ was dissolved in $\mathrm{CH}_{2} \mathrm{Cl}_{2}(10 \mathrm{ml})$, and cooled to $0{ }^{\circ} \mathrm{C}$, $\mathrm{BBr}_{3}(0.88 \mathrm{~mL}, 8 \mathrm{mmol})$ was added dropwise at that temperature and then the solution was stirred at room temperature for $12 \mathrm{~h}$. Water $(50 \mathrm{~mL})$ was added dropwise to quench the reaction. The mixture was extracted 
with ethyl acetate $(3 \times 50 \mathrm{~mL})$. The combined organic layer was dried over anhydrous $\mathrm{Na}_{2} \mathrm{SO}_{4}$ and filtrated. The solvent was evaporated in vacuo. The residue was purified by column chromatography (petroleum ether : ethyl acetate = $5: 1)$. Yield: $176 \mathrm{mg}, 95 \%$; white solid. ${ }^{1} \mathrm{H}$ NMR (400 MHz, $\left.\mathrm{CDCl}_{3}\right), \delta(\mathrm{ppm}): 7.51-7.49(\mathrm{~d}, J$ $=8.8 \mathrm{~Hz}, 2 \mathrm{H}, 2 \mathrm{ArH}), 7.23-7.19(\mathrm{~m}, 2 \mathrm{H}, 2$ thiopheneH), 7.06-7.04 (m, $1 \mathrm{H}$, thiopheneH), 6.86-6.84 (d, $J=$ $8.8 \mathrm{~Hz}, 2 \mathrm{H}, 2$ ArH), 4.96-4.92 (m, 1 H, OH).

\section{Compound 7:}

Compound 5 (176 mg, $1 \mathrm{mmol}$ ) was dissolved in DMF (15 ml), $\mathrm{K}_{2} \mathrm{CO}_{3}(690 \mathrm{mg}, 5 \mathrm{mmol})$, compound 6 (680 $\mathrm{mg}, 1 \mathrm{mmol}$ ) was added and the mixture was stirred at $90{ }^{\circ} \mathrm{C}$ for $12 \mathrm{~h}$. After the reaction was complete (TLC), ethyl acetate $(50 \mathrm{~mL})$ was added, and the mixture was washed with brine $(3 \times 20 \mathrm{~mL})$ and dried over anhydrous $\mathrm{Na}_{2} \mathrm{SO}_{4}$. The solvent was evaporated in vacuo. The residue was purified by column chromatography (petroleum ether : ethyl acetate $=7: 1$ ). Yield: $713 \mathrm{mg}, 87 \%$; white solid. ${ }^{1} \mathrm{H}$ NMR (400 $\left.\mathrm{MHz}, \mathrm{CDCl}_{3}\right), \delta$ (ppm): 7.55-7.53 (d, J= 8.8 Hz, 2 H, 2 ArH), 7.22-7.20 (m, 2 H, 2 thienpenH), 7.07-7.04 (m, $1 \mathrm{H}$, thienpenH), 7.00-6.98 (d, J=8.4 Hz, 2 H, 2 ArH), 6.63 (s, 2 H, 2 ArH), 4.97 (s, 2 H, ArCH $\mathbf{H}_{2} \mathrm{OAr}$ ), 4.00-3.93 (m, 6 H, 3 $\left.\operatorname{ArOCH}_{2}\right), 1.83-1.73\left(\mathrm{~m}, 6 \mathrm{H}, 3 \mathrm{ArOCH}_{2} \mathrm{CH}_{2}\right), 1.48-1.43\left(\mathrm{~m}, 6 \mathrm{H}, 3 \mathrm{ArOCH}_{2} \mathrm{CH}_{2} \mathrm{CH}_{2}\right), 1.27$ (s, $\left.48 \mathrm{H}, 24 \mathrm{CH}_{2}\right)$, 0.90-0.87 (t, $\left.J=6.8 \mathrm{~Hz}, 9 \mathrm{H}, 3 \mathrm{CH}_{3}\right)$.

\section{Compound 8:}

Compound 7 (713 mg, 0.87mmol) was dissolved in $\mathrm{CH}_{3} \mathrm{COOH}(5 \mathrm{ml})$ and $\mathrm{CHCl}_{3}(15 \mathrm{ml})$. Under light exclusion N-Iodosuccinimide (NIS) (292 mg, $1.3 \mathrm{mmol}$ ) was slowly added. The mixture was stirred at room temperature for $12 \mathrm{~h}$. After the reaction was complete (TLC), aqueous saturated $\mathrm{NaHCO}_{3}(30 \mathrm{~mL})$ was slowly added dropwise. The organic layer was separated. The aqueous layer was repeatedly extracted with ethyl acetate $(3 \times 20 \mathrm{~mL})$ and the combined organic layers were washed with brine $(3 \times 20 \mathrm{~mL})$ and dried over anhydrous $\mathrm{Na}_{2} \mathrm{SO}_{4}$, then the solvent was removed in vacuo. The residue was purified by column chromatography (petroleum ether : ethyl acetate $=7: 1$ ). Yield: $707 \mathrm{mg}, 86 \%$; yellow solid. ${ }^{1} \mathrm{H} \mathrm{NMR}\left(\mathrm{CDCl}_{3}\right.$, $400 \mathrm{MHz}): \delta=7.45-7.43$ (d, $J=8.1 \mathrm{~Hz}, 2 \mathrm{H}, 2 \mathrm{ArH}), 7.19$ (d, $J=4 \mathrm{~Hz}, 1 \mathrm{H}$, thiopheneH), 6.98-6.96 (d, $J=$ 8. 2 Hz, 2 H, 2 ArH), 6.87-6.86 (d, J=4 Hz, 1 H, thiopheneH), 6.61 (s, 2 H, 2 ArH), 4.96 (s, 2 H, ArCH 2 OAr),

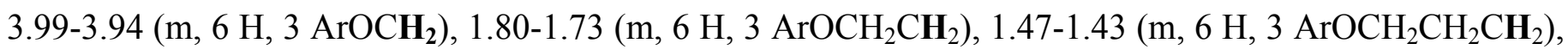
$1.26\left(\mathrm{~s}, 48 \mathrm{H}, 24 \mathrm{CH}_{2}\right), 0.9-0.87\left(\mathrm{t}, J=6.8 \mathrm{~Hz}, 9 \mathrm{H}, 3 \mathrm{CH}_{3}\right)$.

\section{General procedures for the synthesis of FTQ:}


To a $100 \mathrm{~mL}$ round-bottom flask was added compound $3(77 \mathrm{mg}, 0.34 \mathrm{mmol})$ and compound 8 (707 $\mathrm{mg}, 0.75$ mmol) was dissolved in dry THF $(10 \mathrm{~mL})$ and dry triethylamine $(5 \mathrm{~mL})$ under a nitrogen atmosphere. Then $\mathrm{Pd}\left(\mathrm{PPh}_{3}\right)_{2} \mathrm{Cl}_{2}(15 \mathrm{mg}), \mathrm{CuI}(12 \mathrm{mg}, 0.08 \mathrm{mmol})$ was added, and the mixture was refluxed at $50{ }^{\circ} \mathrm{C}$ for $15 \mathrm{~h}$. After the reaction was complete (TLC), ethyl acetate $(20 \mathrm{~mL})$ was added, and the mixture was washed with brine $(3 \times 20 \mathrm{~mL})$ and dried over anhydrous $\mathrm{Na}_{2} \mathrm{SO}_{4}$. The solvent was evaporated in vacuo. The residue was purified by column chromatography (petroleum ether : Methylene chloride =3:1). Yield: $298 \mathrm{mg}, 47 \%$; red solid. ${ }^{1} \mathrm{H}$ NMR (300 MHz, $\mathrm{CDCl}_{3}$ ), $\delta$ (ppm): 7.93 (d, $2 \mathrm{H}, J=1.2 \mathrm{~Hz}$, fluorenone-1,8H), 7.75-7.72 (dd, $J=$ $1.8 \mathrm{~Hz}, J=7.8 \mathrm{~Hz}, 2 \mathrm{H}$, fluorenone-4,5H), 7.58-7.55 (d, $J=8.7 \mathrm{~Hz}, 4 \mathrm{H}, 4 \mathrm{ArH}), 7.54-7.51$ (d, J= 7.8 Hz, 2 H, fluorenone-3,6H), 7.36-7.34 (d, $J=3.6 \mathrm{~Hz}, 2 \mathrm{H}$, thiopheneH), 7.21-7.20 (d, $J=3.9 \mathrm{~Hz}, 2 \mathrm{H}, 2$ thiopheneH), 7.03-7.00 (d, $J=8.7$ Hz, 4 H, 4 ArH), 6.63 (s, 4 H, 4 ArH), 4.99 (s, 4 H, 2 OCH ), 4.01-3.93 (m, 12 H, 6 OCH 2$), 1.84-1.70\left(\mathrm{~m}, 12 \mathrm{H}, 6 \mathrm{OCH}_{2} \mathrm{CH}_{2}\right), 1.47$ (m, $\left.12 \mathrm{H}, 6 \mathrm{OCH}_{2} \mathrm{CH}_{2} \mathrm{CH}_{2}\right), 1.25$ (m, $\left.96 \mathrm{H}, 48 \mathrm{CH}_{2}\right), 0.90$ $0.85\left(\mathrm{t}, J=6.6 \mathrm{~Hz}, 18 \mathrm{H}, 6 \mathrm{CH}_{3}\right) .{ }^{13} \mathrm{C} \mathrm{NMR}\left(100 \mathrm{MHz}, \mathrm{CDCl}_{3}\right): \delta=193.30,158.70,153.38,144.29,142.60$, 141.22, 138.16, 135.34, 135.14, 131.65, 131.15, 127.16, 126.94, 124.62, 123.15, 121.07, 120.73, 115.37, 106.23, 93.10, 86.07, 73.46, 70.56, 69.22, 31.94, 30.38-29.38 (multi carbons in alkyl chain), 26.17, 26.14, 22.70, 14.10; Elemental analysis calcd (\%) for $\mathrm{C}_{123} \mathrm{H}_{176} \mathrm{O}_{9} \mathrm{~S}_{2}$ (1862.87): C, 79.31; H, 9.52. found: C, 76.98; H, 9.27; HRMS (ESI): m/z calcd for $\mathrm{C}_{123} \mathrm{H}_{176} \mathrm{O}_{9} \mathrm{~S}_{2} \mathrm{Na}[\mathrm{M}+\mathrm{Na}]^{+} 1885.28$, found 1885.28.

\section{2. ${ }^{1} \mathrm{H}$ and ${ }^{13} \mathrm{C}$ NMR spectra for representative compounds}

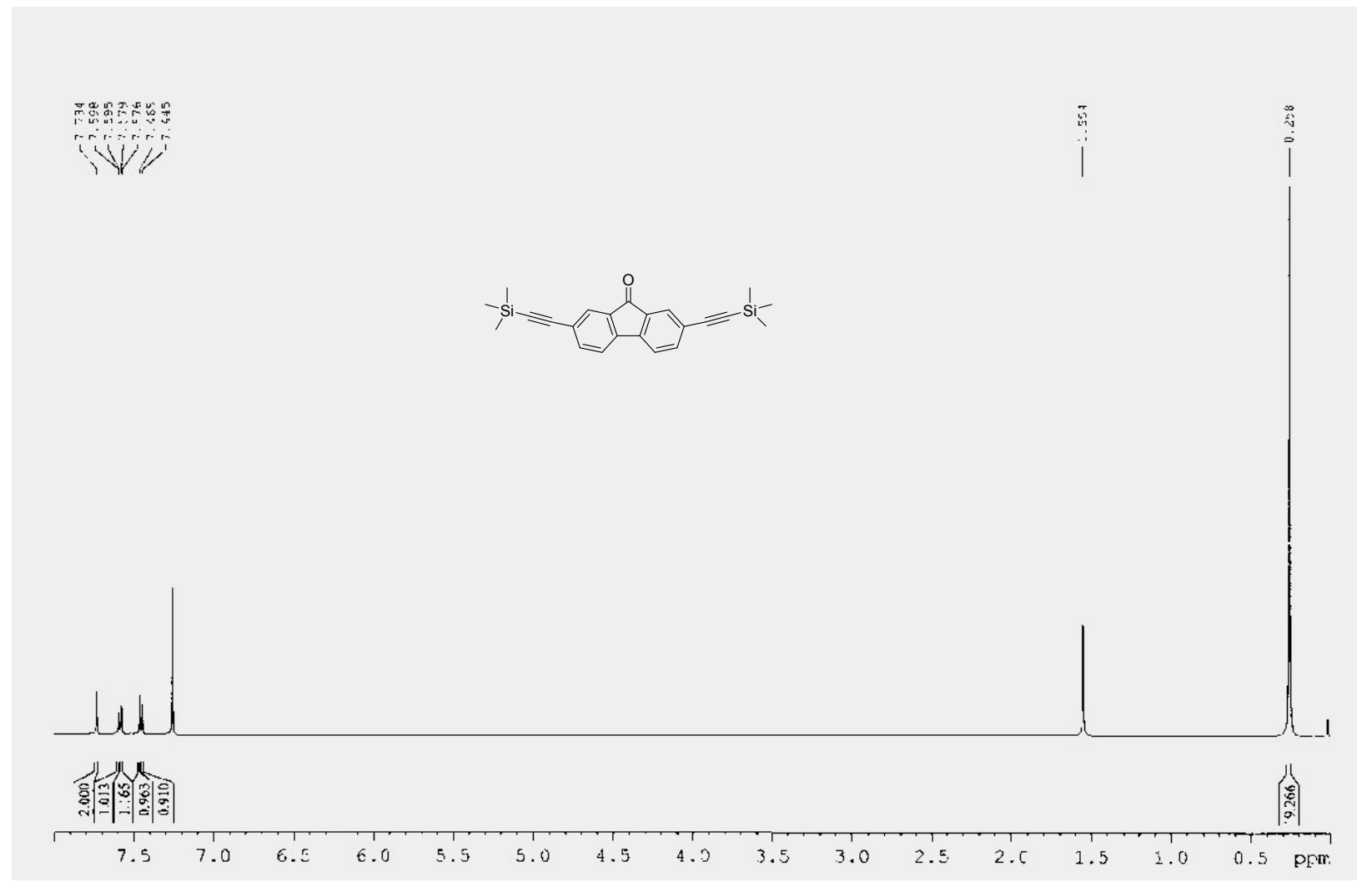


${ }^{1} \mathrm{H} \mathrm{NMR}\left(\mathrm{CDCl}_{3}, 400 \mathrm{MHz}\right.$ ppm) spectra of compound 2.

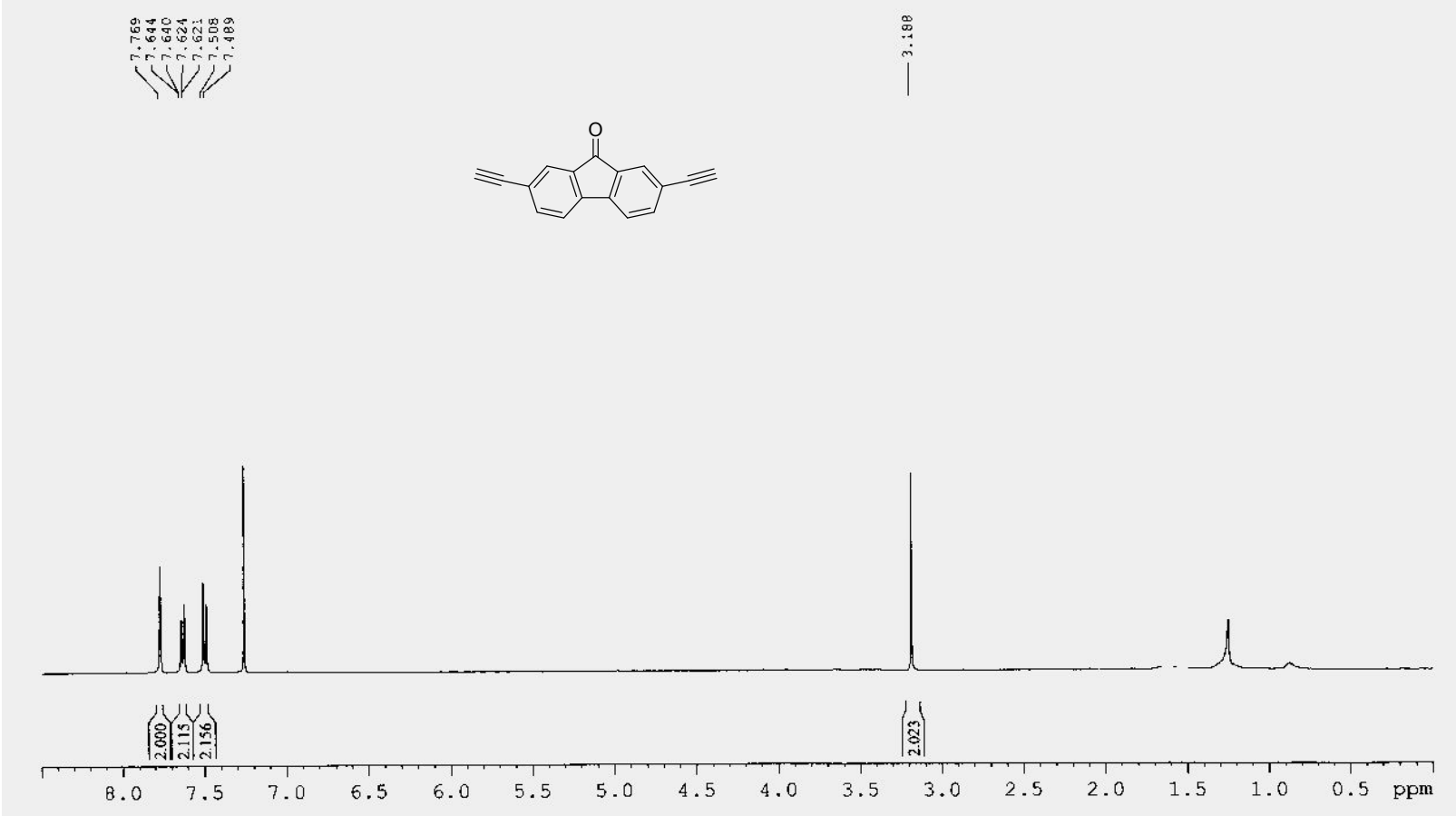

${ }^{1} \mathrm{H} \mathrm{NMR}\left(\mathrm{CDCl}_{3}, 400 \mathrm{MHz} \mathrm{ppm}\right)$ spectra of compound 3.

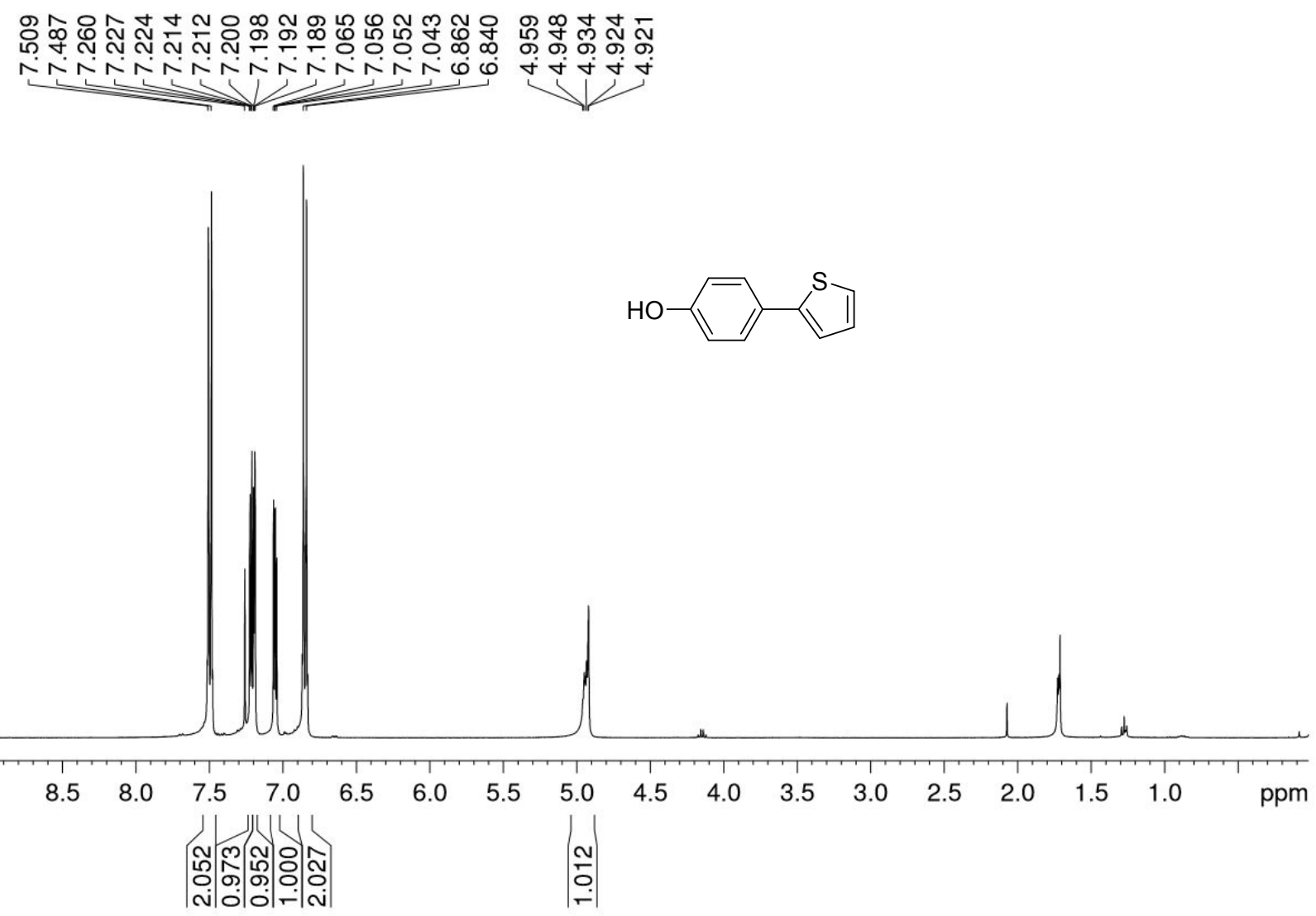


${ }^{1} \mathrm{H} \mathrm{NMR}\left(\mathrm{CDCl}_{3}, 400 \mathrm{MHz} \mathrm{ppm}\right)$ spectra of compound 5.

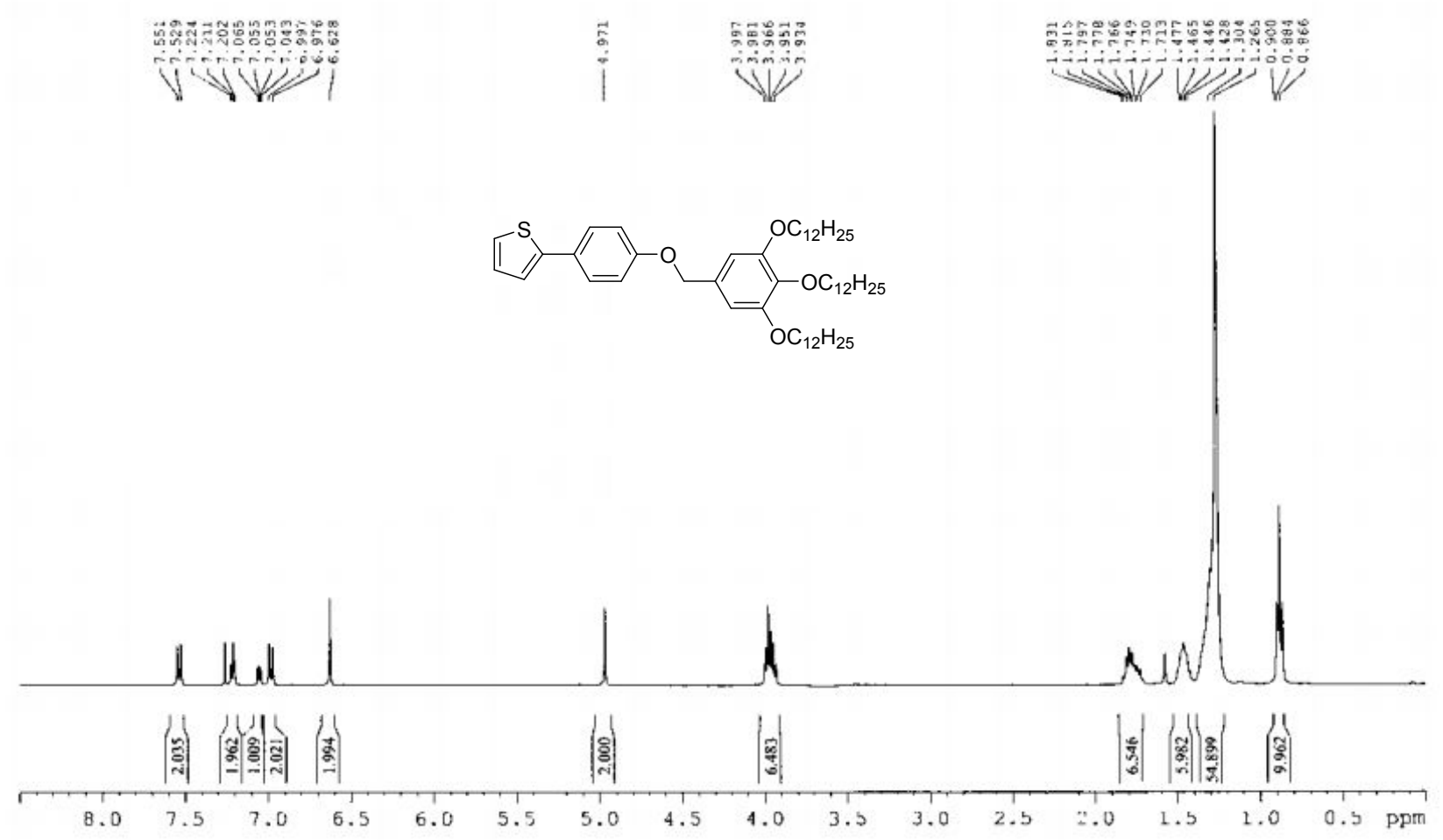

${ }^{1} \mathrm{H} \mathrm{NMR}\left(\mathrm{CDCl}_{3}, 400 \mathrm{MHz} \mathrm{ppm}\right)$ spectra of compound 7. 


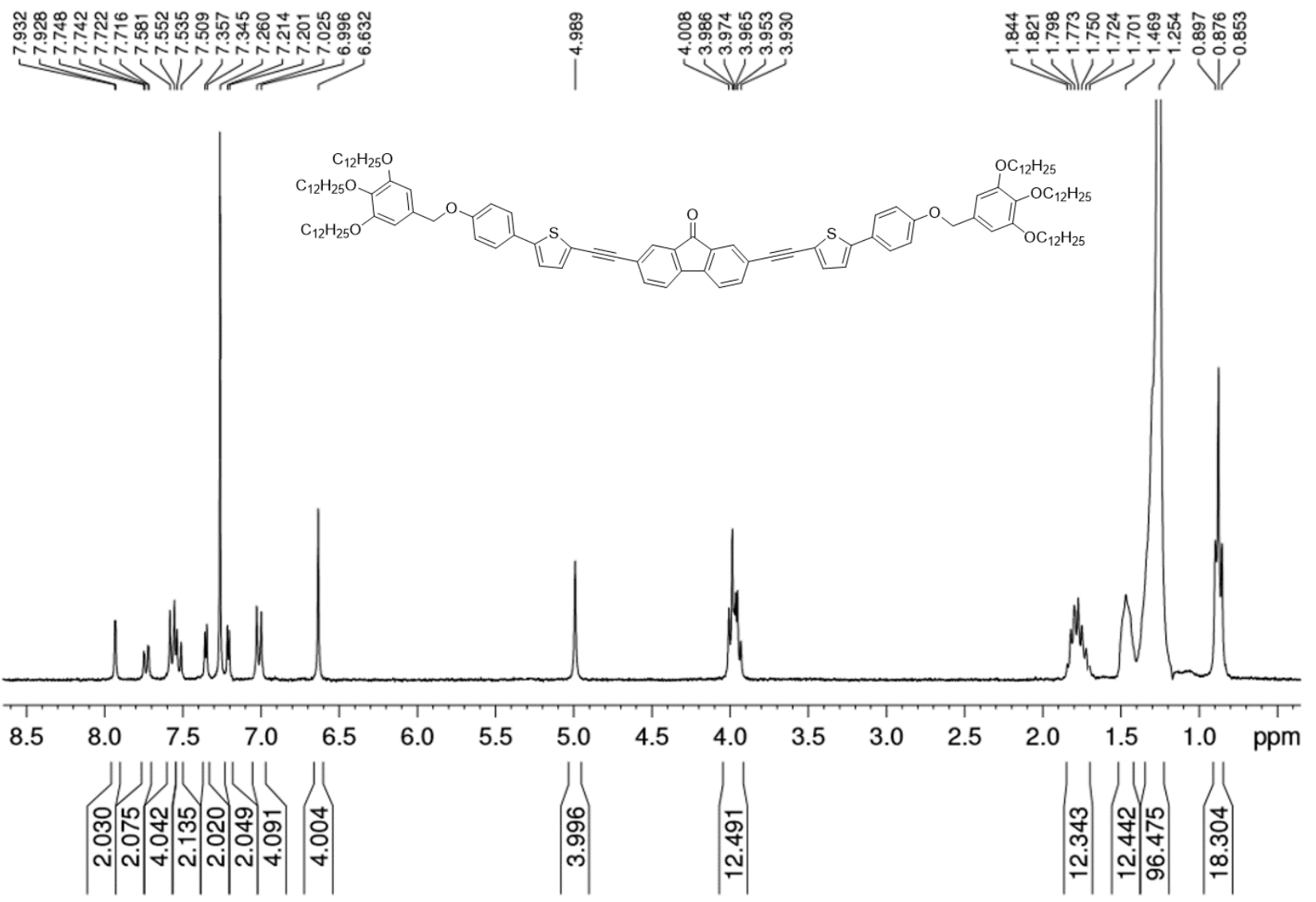

${ }^{1} \mathrm{H} \mathrm{NMR}\left(\mathrm{CDCl}_{3}, 300 \mathrm{MHz}\right.$ ppm) spectra of compound FTQ.

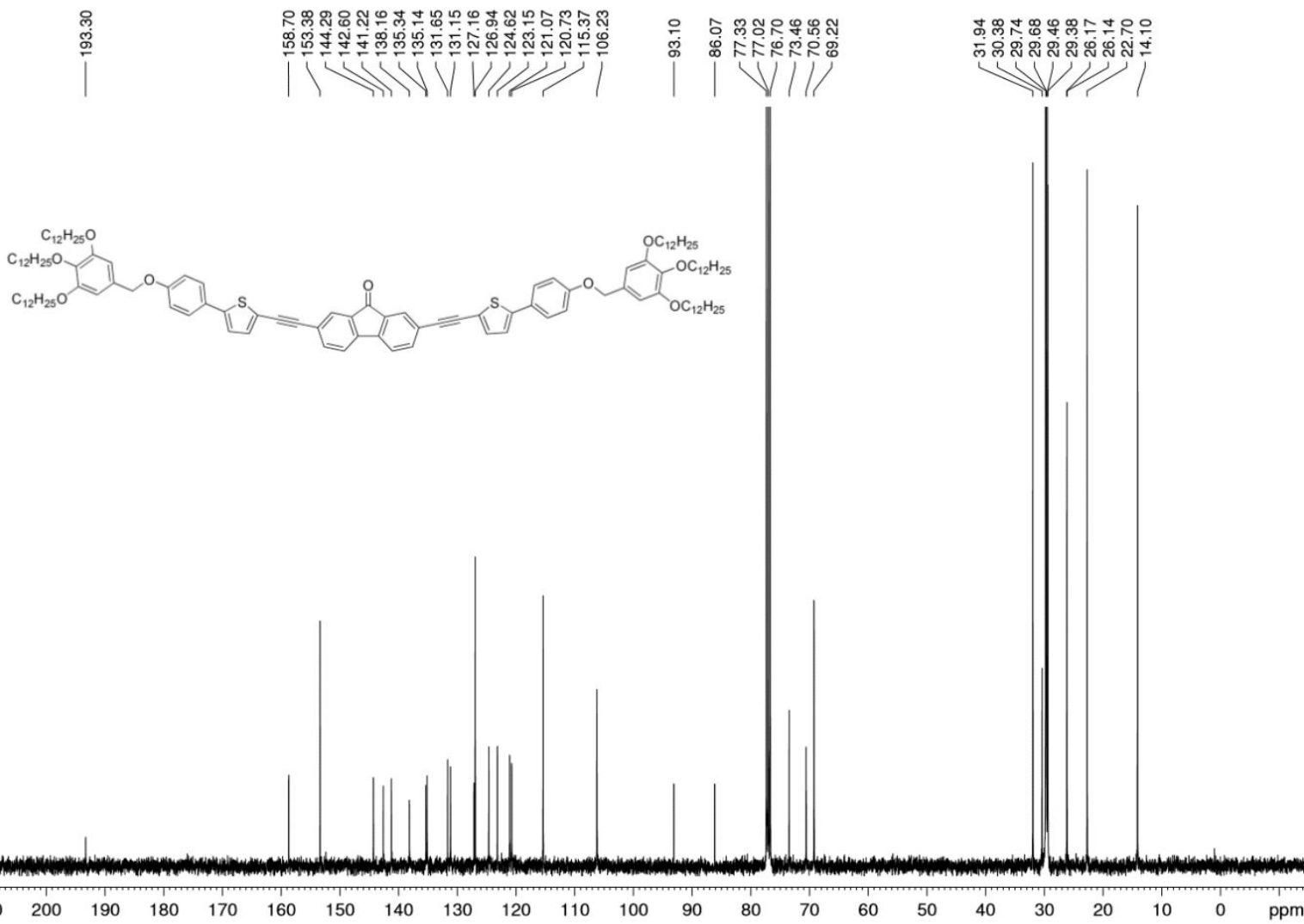

${ }^{13} \mathrm{C} \mathrm{NMR}\left(\mathrm{CDCl}_{3}, 100 \mathrm{MHz}, \mathrm{ppm}\right)$ spectra of compound FTQ.

\section{SAXS reflection of FTQ at $90{ }^{\circ} \mathrm{C}$}




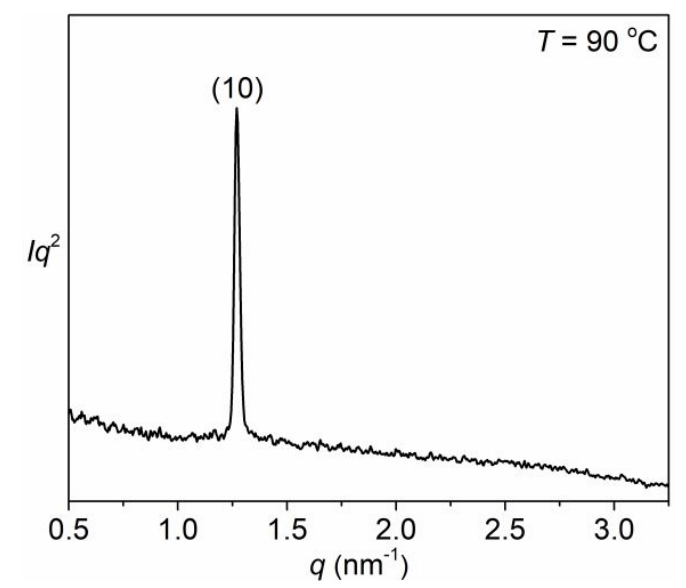

Figure S1. SAXS pattern of FTQ at $T=90^{\circ} \mathrm{C}$.

(1) $\mathrm{Col}_{\text {hex }} / p 6 m m:$

Table S1 Experimental and Calculated $\boldsymbol{d}$-Spacing of the Observed SAXS Reflection of the Hexagonal Columnar Phase in FTQ at $90^{\circ} \mathrm{C}$

$d_{\text {obs. }}-$ spacing

$(\mathrm{nm})$

4.95 $d_{\text {cal. }}$ - spacing

(nm)

4.95 intensity

100.0
Phase

0

(20)

$$
a_{\text {hex }}=5.72 \mathrm{~nm}
$$

All intensity values are Lorentz and multiplicity corrected.

(2) $\mathrm{Col}_{\text {squ }} / p 4 m m:$

Table S2 Experimental and Calculated $\boldsymbol{d}$-Spacing of the Observed SAXS

Reflection of the Square Columnar Phase in FTQ at $90^{\circ} \mathrm{C}$

\begin{tabular}{ccccc}
\hline$(h k)$ & $\begin{array}{c}d_{\text {obs. }}-\text { spacing } \\
(\mathrm{nm})\end{array}$ & $\begin{array}{c}d_{\text {cal. }}-\text { spacing } \\
(\mathrm{nm})\end{array}$ & intensity & Phase \\
\cline { 1 - 4 }$(10)$ & 4.95 & 4.95 & 100.0 & 0 \\
$(11)$ & & & \\
$(20)$ & & \\
\hline \multicolumn{5}{c}{$a_{\text {squ }}=4.95 \mathrm{~nm}$} \\
\hline
\end{tabular}

All intensity values are Lorentz and multiplicity corrected. 


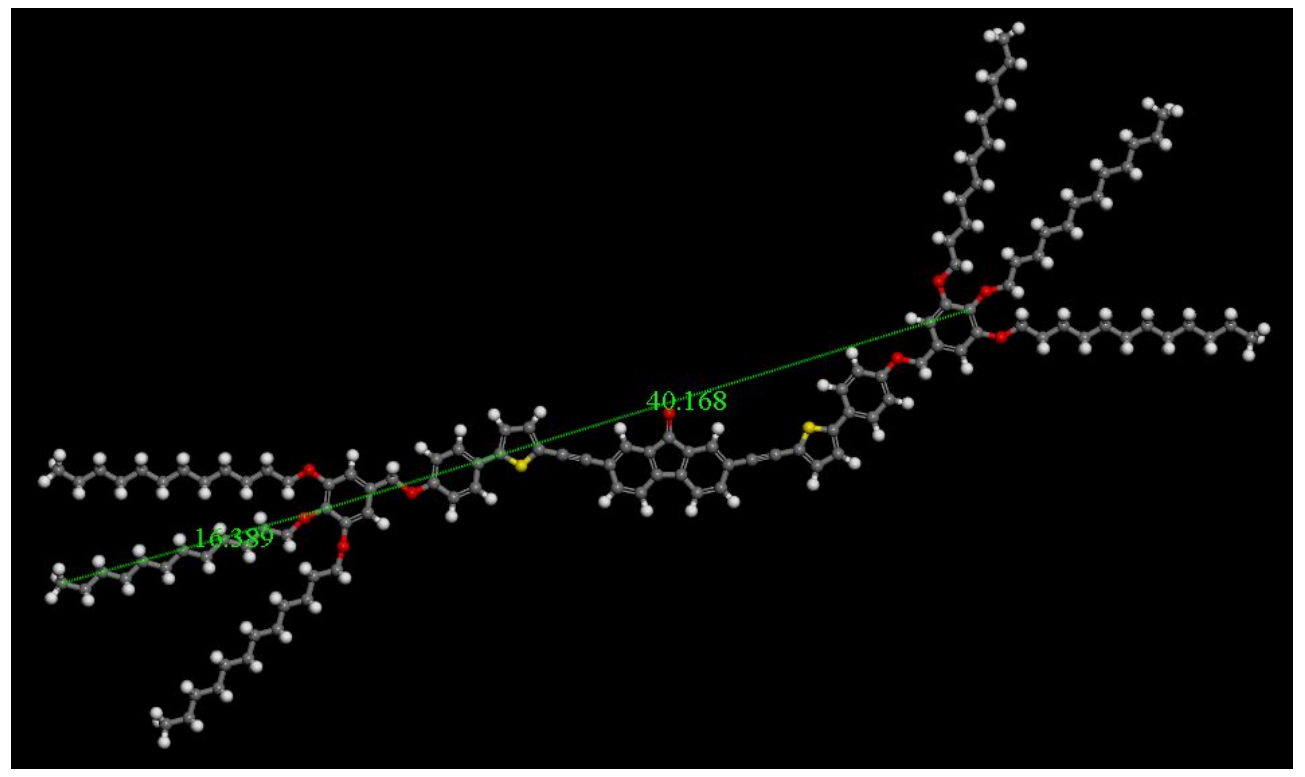

Figure S2. The calculated lattice parameter according to the size of FTQ in Material Studio: $4.02+1.64=$ $5.66 \mathrm{~nm}$.

\section{DFT calculations}
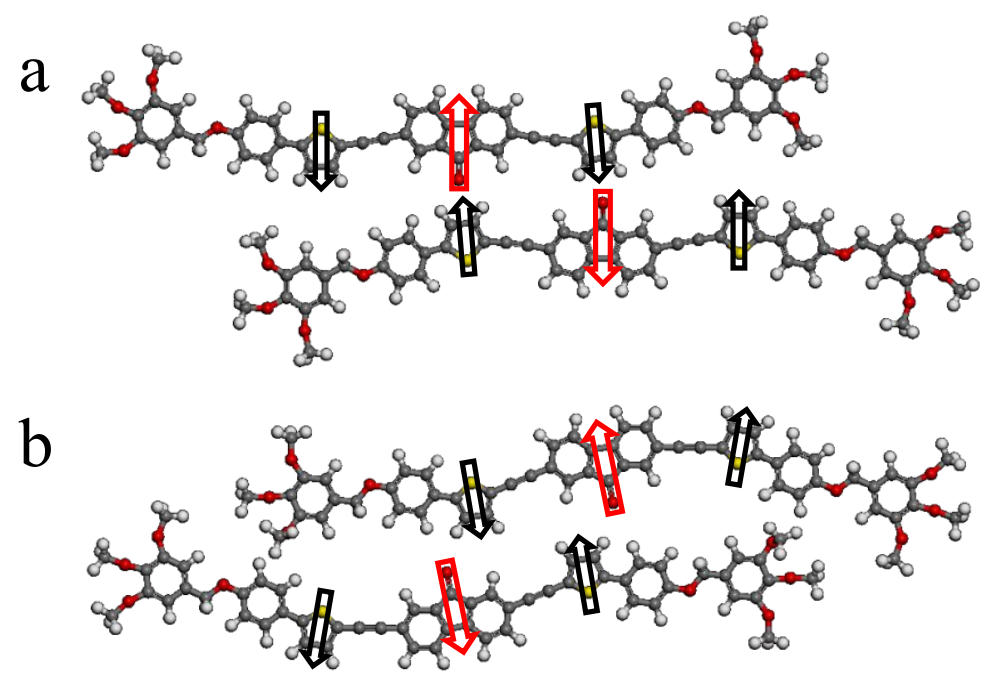

Figure S3. The intermolecular dipole-dipole interactions of cis-conformation and trans-conformation. 


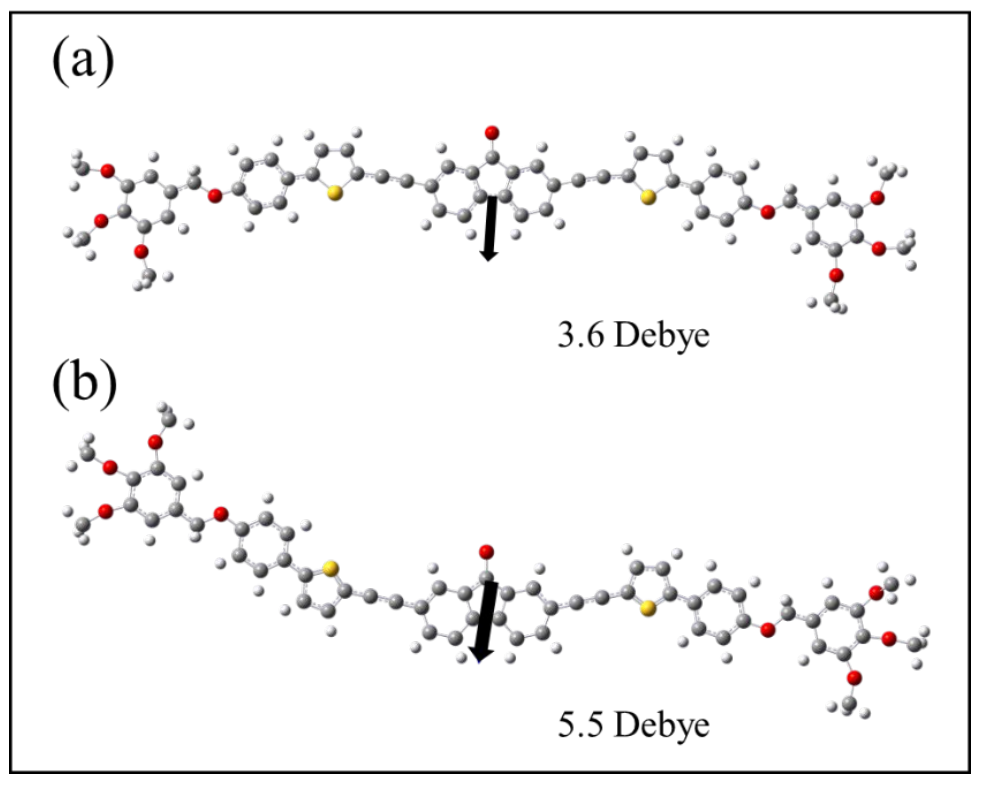

Figure S4. Calculated dipole moment of (a) I-shaped conformer and (b) S-shaped conformer

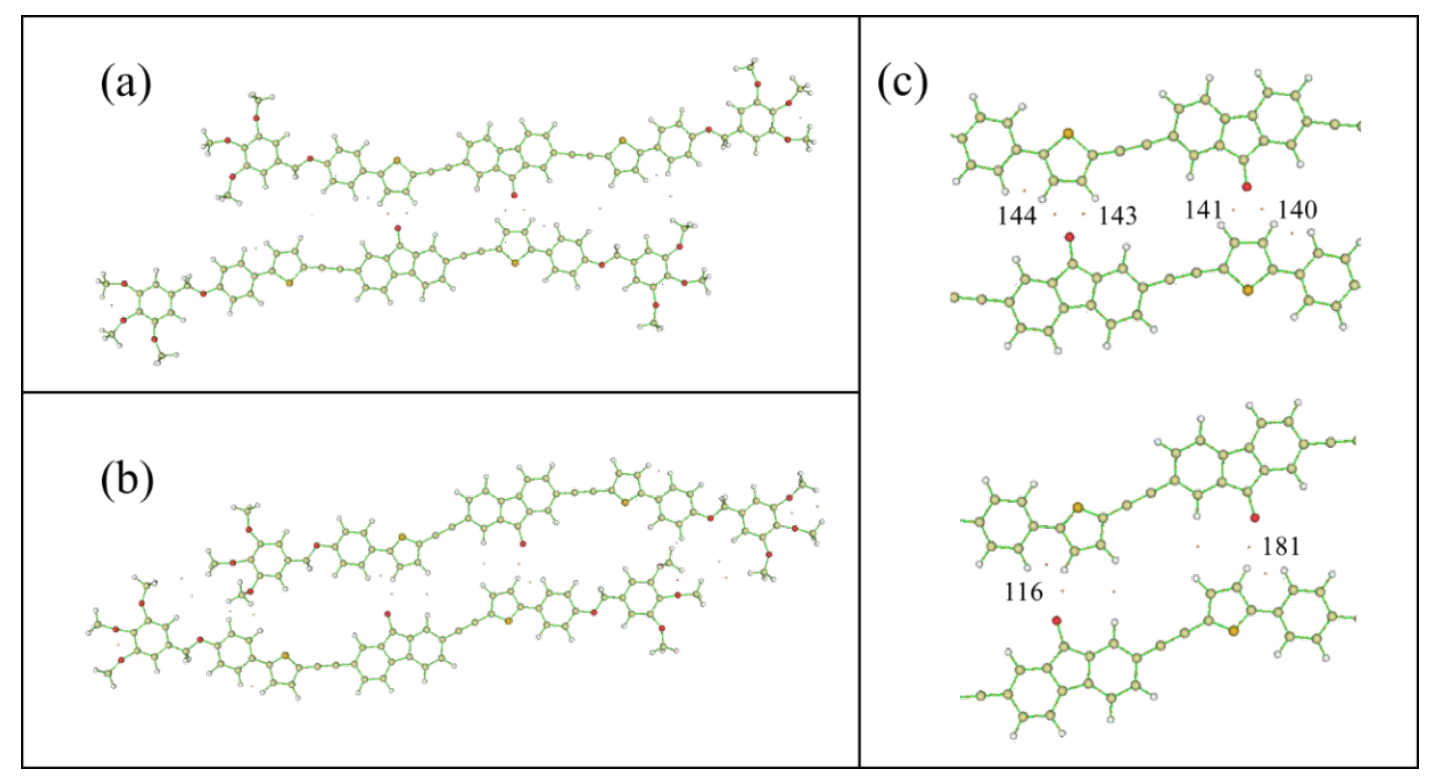

Figure S5. Molecular graphs showing intermolecular bond critical points (BCPs, shown as small orange dots) for various structural dimer motifs of FTQ. (a) dimer-I; (b) dimer-S; (c) the BCPs are shown in the enlarged

S1. Zhao, K.; Xiao, Y.; Guo, C.; Chang, Q.; Gao, H.; Cheng, X. H. Tetrahedron 2019, 75, 409-415.

S2. Byron, D. J.; Komitov, L.; Matharu, A. S.; MCsherry, I.; Wilson, R. C. The synthesis and characterisation of a novel thiophene-based liquid crystal exhibiting ferro-, ferri- and antiferro-electric phase types. Journal of 
Materials Chemistry 1996, 6, 1871-1878.

S3. Fang, H.; Gao, H.; Wang, T.; Zhang, B.; Xing, W.; Cheng, X. H. Benzothiadiazole-based D- $\pi-A-\pi-D$ fluorophores: Synthesis, self-assembly, thermal and photophysical characterization. Dyes and Pigments 2017, 147, 190-198.

S4. Cheng, X.; Su, F.; Huang, R.; Gao, H.; Marko, P.; Carsten, T. Effect of central linkages on mesophase behavior of imidazolium-based rod-like ionic liquid crystals. Soft matter 2012, 8, 2274-2285. 